\title{
Microprocessor control system for a robotic platform for automated care for agricultural crops
}

\author{
Artur Dyshekov ${ }^{1, *}$, Maxim Mirzaev ${ }^{1}$, Madin Shereuzhev ${ }^{2}$, and Artem Peshnin ${ }^{3}$ \\ ${ }^{1}$ Federal Scientific Agro Engineering Center VIM, 109428, 1st Institutsky proezd, 5, Moscow, Russia \\ ${ }^{2}$ Moscow State Technical University N.E. Bauman,105005, 2nd Baumanskaya St., 5, building 1, \\ Moscow, Russia \\ ${ }^{3}$ Moscow State Technical University «Stankin», 127055, Vadkovsky lane, 1, Moscow, Russia
}

\begin{abstract}
In this paper, the use of electronic components for automating the care of agricultural crops is studied. A microprocessor control system has been developed, components of a robotic platform have been selected to ensure stable operation of the complex in the field. This platform combines ease of use and high performance. At the moment, there are few analogous foreign complexes with similar characteristics, therefore, the considered robotic platform is a promising development.
\end{abstract}

\section{Introduction}

One of the ways to increase the productivity and quality of agricultural products is to automate production processes [7]. By using robotic systems, it is possible to increase the speed of weeding, planting, and delivery of fertilizers to agricultural crops. To complete the task, the robotic complex must have sufficient positioning accuracy for the tool.

To achieve the above goal, a robotic complex was developed that can perform a number of agrotechnical tasks, such as planting, watering, fertilizing, loosening, removing weeds, harvesting. Each of the operations is configured to be performed automatically. The complex consists of aluminum structural parts, on which a standard mount is provided for replaceable modules of the working body for each operation. The soil module is a special substrate for the soil. The complex is equipped with a phytolamp with controlled lighting brightness.

The complex has a working area of $400 \times 300 \times 30 \mathrm{~mm}$. The set provides the ability to move the elements of the working body in three axes OX, OY, OZ within the work area. Stepper motors [1][8] are used for this purpose.

The control component of the plotter is a board with built-in support for USB connection to a computer.

The technical task of the complex is to automate the processes of planting, watering, weeding of crops, depending on the installed tool. The schematic diagram of a microprocessor system consists of a microcomputer[12][15], stepper motor controllers[3],

\footnotetext{
* Corresponding author: a.i.dyshekov@gmail.com
} 
stepper motors, three limit switches, a mechanical attachment, which is selected depending on the task being performed.

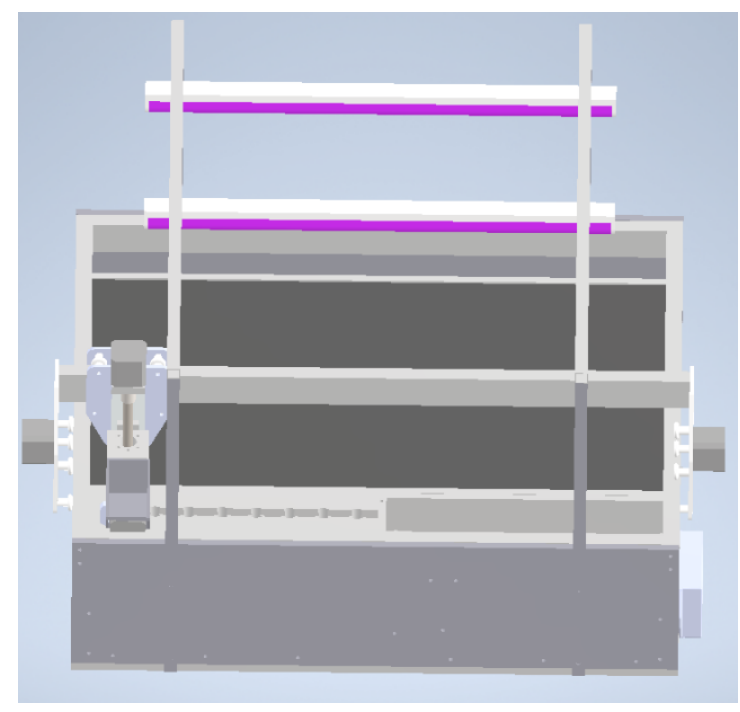

Fig. 1. 3d model of the complex.

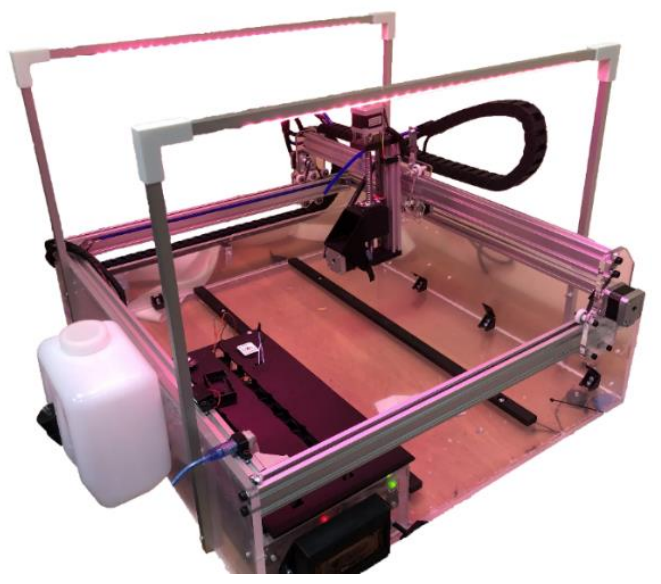

Fig. 2. Appearance of the complex.

\section{Choice of component base}

The Arduino Mega 2560 microcontroller is used as a computing module. This microcontroller allows you to transmit a signal to the stepper motor controllers, to control the instrument installed on the device. 


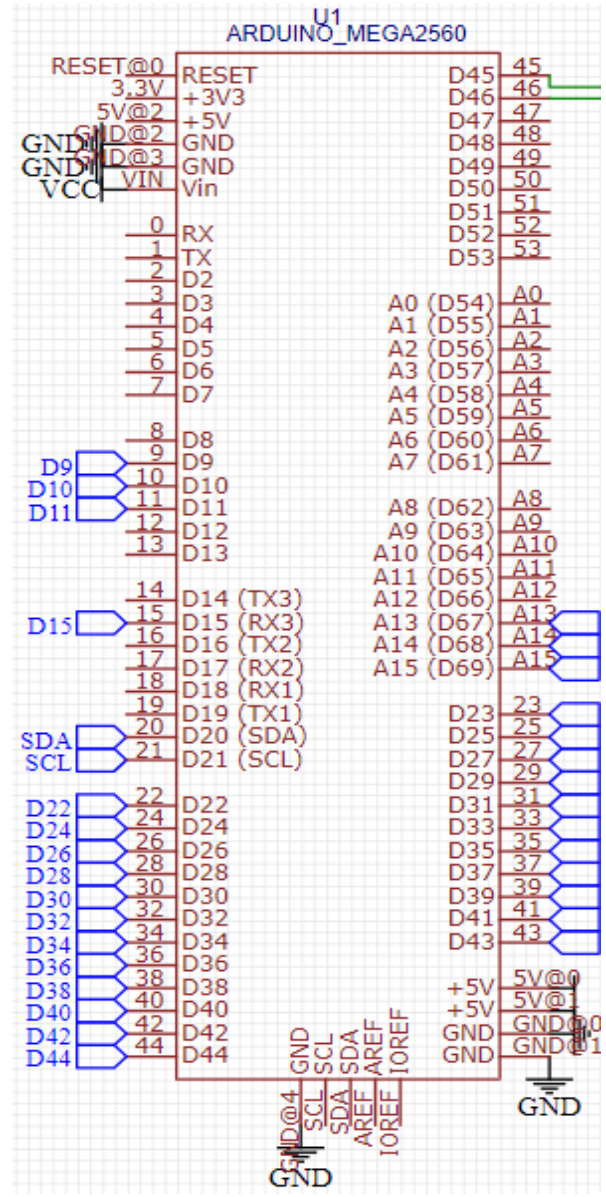

Fig. 3. Schematic designation of the controller Arduino mega 2560.

To simplify the connection of all system components to the controller, a board in the Arduino Mega 2560 form factor was developed. To ensure the autonomous operation of the complex, there is a DC-DC step-down converter on the board from 12 to 5 volts. It supplies power to the controller.

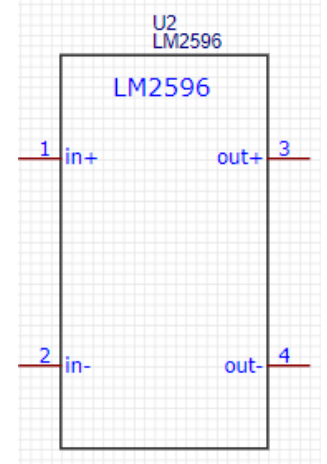

Fig. 4. Schematic designation of DC - DC converter LM2596. 


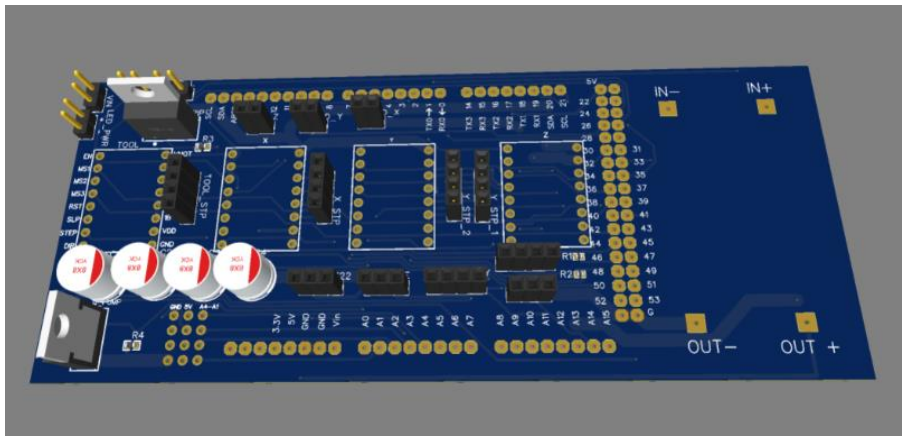

Fig. 5. External view of the developed board.

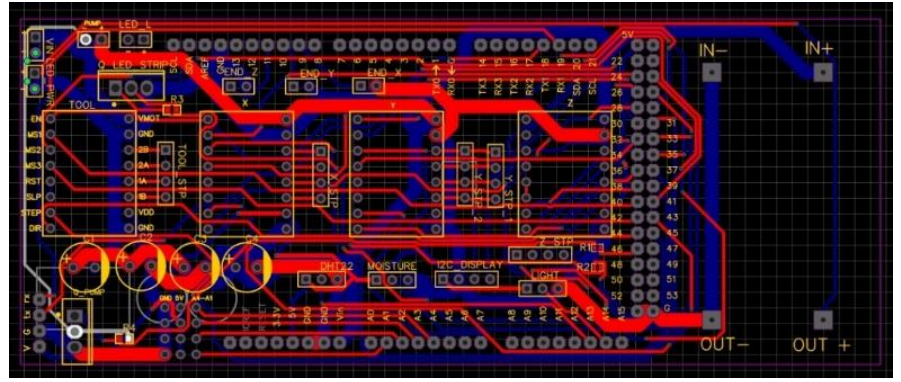

Fig. 6. Board layout.

Also, the developed board contains an N-Channel power switch designed to control the backlight and pump of the complex.

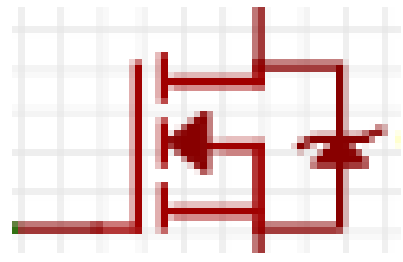

Fig. 7. Schematic designation of the N-Channel power switch.

The movement of the carriage with the tool along the $\mathrm{X}$ and $\mathrm{Y}$ axes is carried out using a belt drive. The Z-axis is driven by a ball screw. Stepper motors of the NEMA 17 form factor are used to drive the mechanical part.

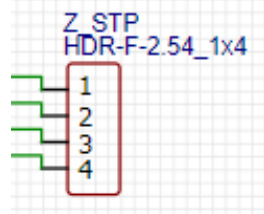

Fig. 8. Schematic designation of NEMA-17 stepper motors.

Pololu A4988 modules act as stepper motor controllers. With their help, you can set the direction and speed of movement of stepper motors. 


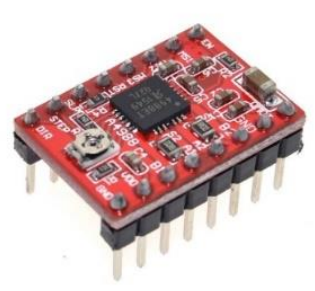

Fig. 9. Exterior view of the Pololu A4988 stepper motor controller.

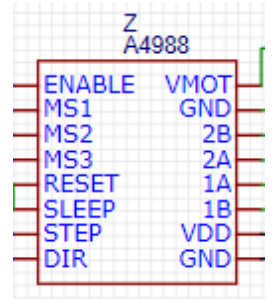

Fig. 10. Schematic designation of the Pololu A4988 stepper motor controller.

To determine the starting position of the carriage with the tool, limit switches are installed on all axes. Before starting work, the carriage moves to the starting position.

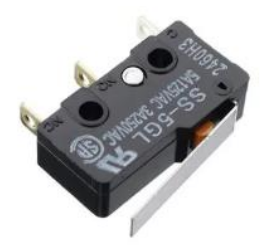

Fig. 11. External view of the limit switch.

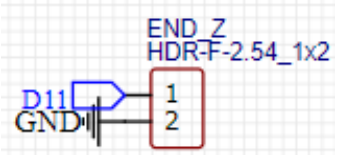

Fig. 12. Schematic designation of the limit switch.

To read data on the environment, the complex is equipped with sensors [4] for temperature, air humidity, soil moisture and illumination. Their readings are processed on a microcontroller and displayed on a liquid crystal i2c [10] display.

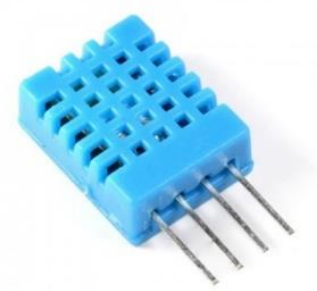

Fig. 13. External view of the temperature and humidity sensor. 


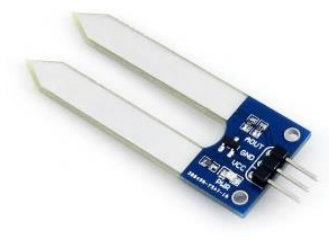

Fig. 14. External view of the soil moisture sensor.

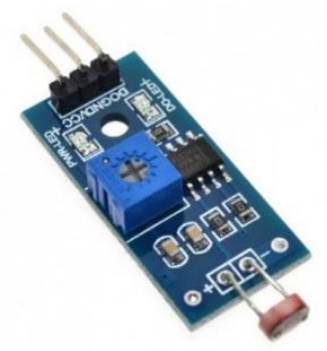

Fig. 15. External view of the light sensor [13].

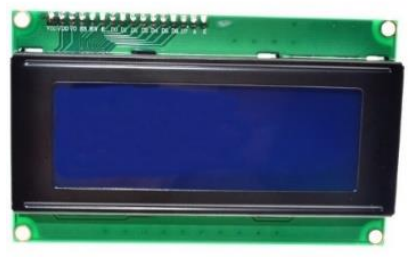

Fig. 16. Display layout.

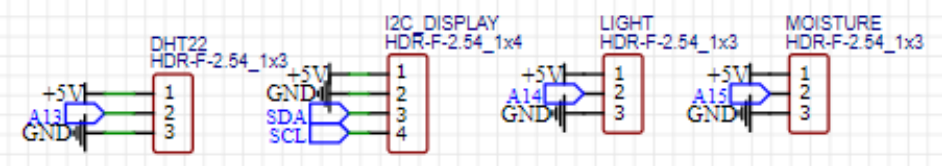

Fig. 17. Schematic view of sensors and display.

Depending on the installed nozzle, the microcontroller sends a corresponding control signal to it. The gripping and drilling device is controlled via the stepper motor controller. The sprayer [6] is controlled by the power switch N-Channel, when using a PWM signal it allows smooth switching of the supplied voltage and control of the sprayer pump [5].

For the most convenient assembly and connection of electronic components of the device, an adapter PCB [9] board was developed. It is attached to the case in such a way that all wires can be connected without hindrance without disassembling the case. Connectors for connecting stepper motors, limit switches, display and all sensors are brought out to the adapter board on both sides. From the inside, the board is connected to the controller using wires [11]. 


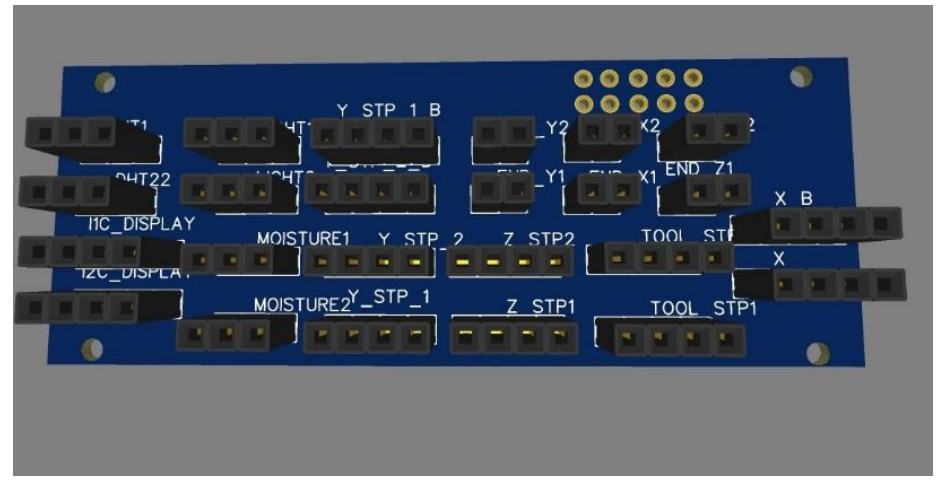

Fig. 18. External view of the adapter board.

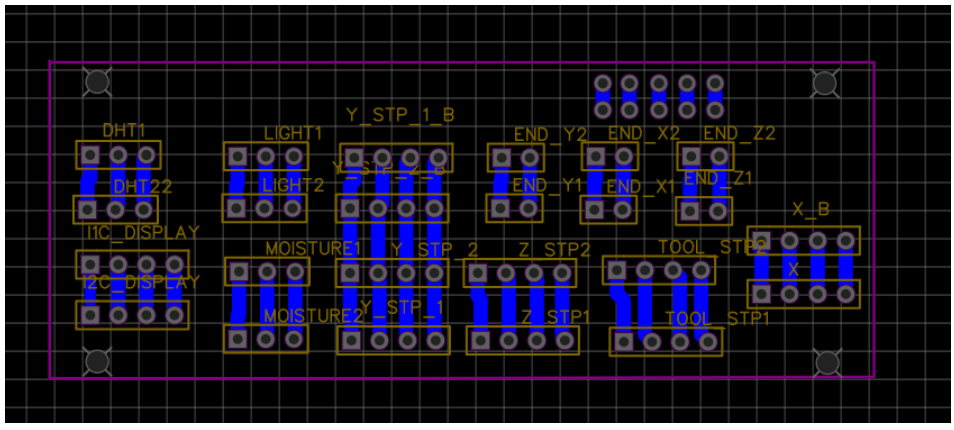

Fig. 19. Scheme of the adapter board.
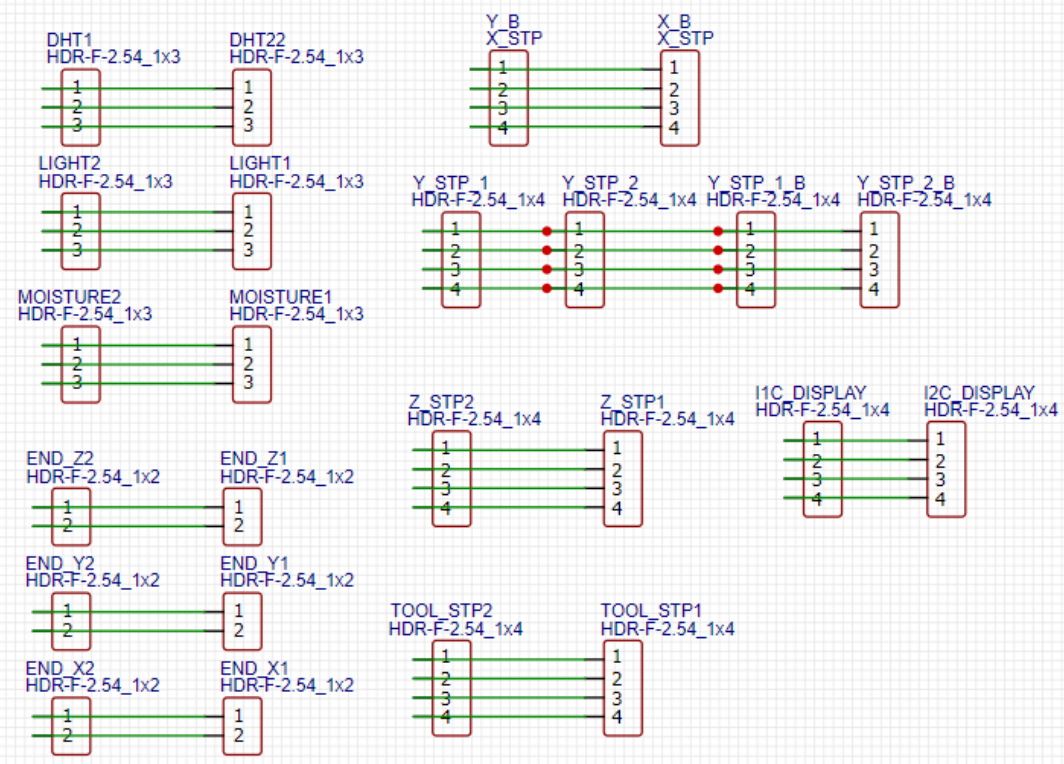

Fig. 20. Schematic diagram of the adapter board. 
Based on the components described above, a schematic diagram of an automatic control system was developed.

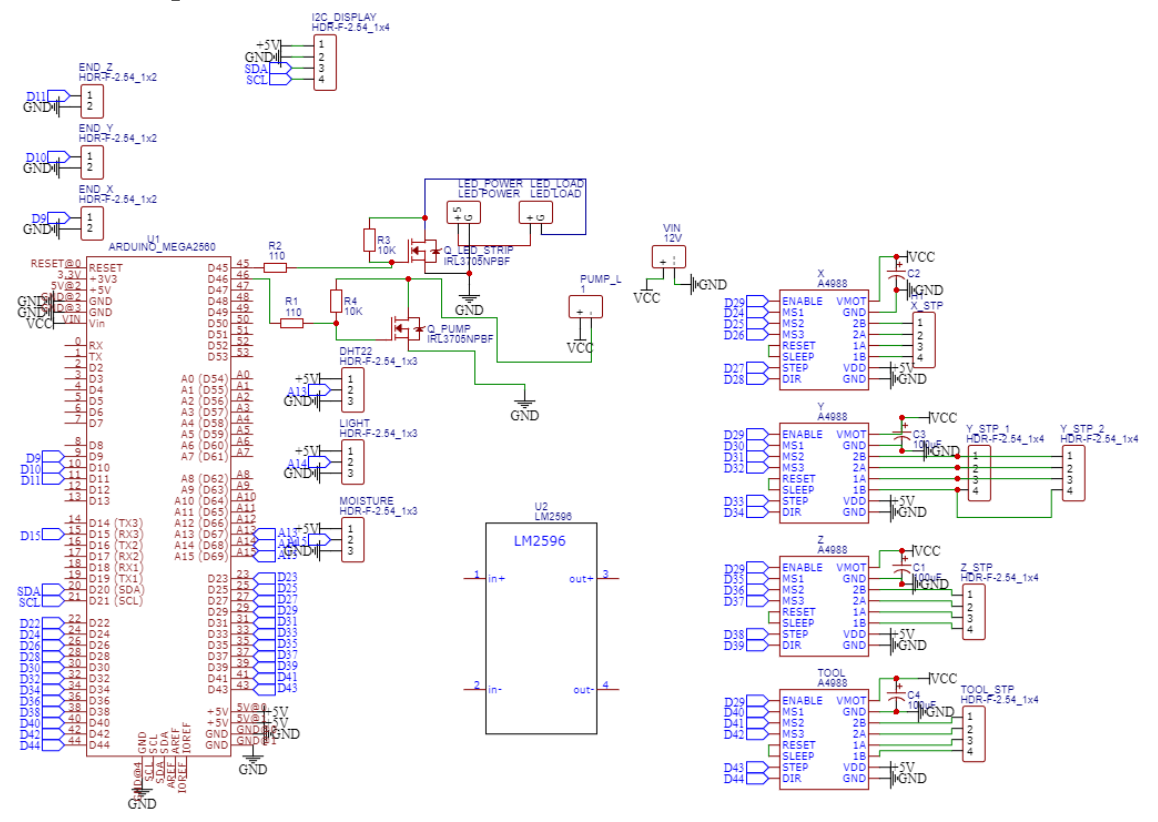

Fig. 21. Schematic diagram of the automatic control system.

\section{Description of interfaces}

The computing module is connected to a personal computer as a peripheral device, and then it is required to load the program into the memory of the computing module. The program contains a sequence of points along which the carriage should move with the installed tool. Points are specified by coordinates along the $\mathrm{X}, \mathrm{Y}, \mathrm{Z}$ axes of the tool. The computing module calculates the trajectory of movement at the given points and transmits the control signal to the stepper motor controllers. Limit switches are used to determine the starting position of the device carriage. Depending on the selected tool (gripper [2][14], drill bit, sprayer), the computing module sends a corresponding control signal to the installed tool.

\section{Research results}

During the research, a board was developed that simplifies the connection of the electronic components of the system to the controller.

The wiring diagram shows all devices and components that make up the product, their input and output elements (connectors, board, etc.).

The logical part of the system being developed is located in a specialized building. It consists of a controller board, stepper motor controllers, a power switch, and two adapter boards. The housing has built-in female connectors for connecting the power section. The power section consists of a power supply unit. 


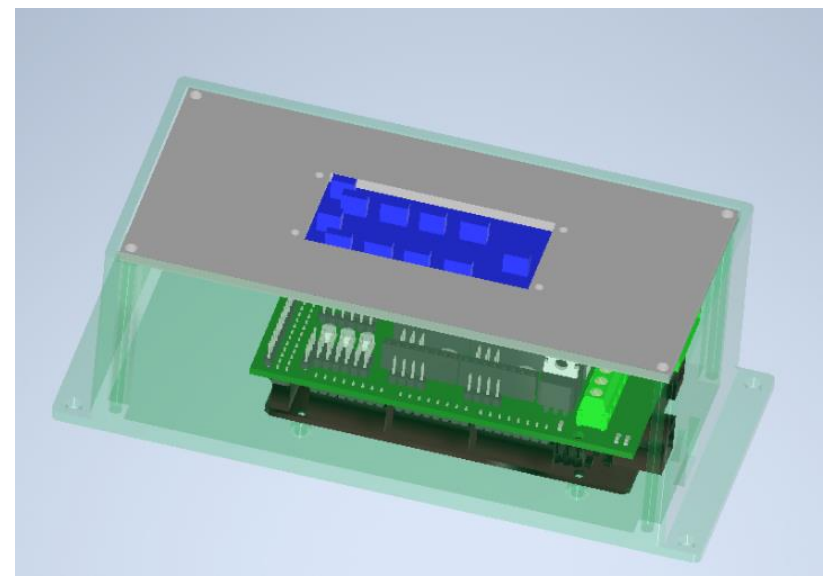

Fig. 22. External view of the case for the logical part of the complex.

\section{Conclusion}

The developed control system of the autonomous robotic complex allows automatic fertilization, watering, and weeding of agricultural crops.

The carriage of this complex can move in three planes for the most accurate positioning of the tool and performing the required action.

In the course of the analysis, the communication interfaces between various devices of the microprocessor system were studied. The requirements for the design and installation of electronic systems, including the logical part and power supply circuits, have been determined. A board has been developed to facilitate connection of all elements of the complex to the controller.

As a result, based on laboratory tests, it can be concluded that a microprocessor system for controlling a robotic complex of autonomous care for agricultural crops is applicable in the field.

\section{References}

1. R. Crowder, Stepper motors, Electric Drives and Electromechanical Systems 978-0-08102884-1, 209-226 (2019), https://doi.org/10.1016/B978-0-08-102884-1.00008-X

2. B. Zhang, Y. Xie, J. Zhou, K. Wang, Zh. Zhang, State-of-the-art robotic grippers, grasping and control strategies, as well as their applications in agricultural robots: A review, Computers and Electronics in Agriculture 0168-1699, 105694 (2020), https://doi.org/10.1016/j.compag.2020.105694

3. Q. Fa-Qun, J.-Dong, Z.Shi-qing, Design of stepping motor control system based on AT89C51 microcontroller, Procedia Engineering 1877-7058, 2276-2280 (2011), https://doi.org/10.1016/j.proeng.2011.08.426

4. A.S. Ali, Z. Zanzinger, D. Debose, B. Stephens, Open Source Building Science Sensors (OSBSS): A low-cost Arduino-based platform for long-term indoor environmental data collection, Building and Environment 0360-1323, 114-126 (2016), https://doi.org/10.1016/j.buildenv.2016.02.010 
5. R. Salcedo, H. Zhu, Zh. Zhang, Zh. Wei, L. Chen, E. Ozkan, D. Falchieri, Foliar deposition and coverage on young apple trees with PWM-controlled spray systems, Computers and Electronics in Agriculture 0168-1699, 105694 (2020), https://doi.org/10.1016/j.compag.2020.105794

6. Zh. Zhang, H. Zhu, H. Guler, Y. Shen, Improved premixing in-line injection system for variable-rate orchard sprayers with Arduino platform, Computers and Electronics in Agriculture 0168-1699, 389-396 (2019), https://doi.org/10.1016/j.compag.2019.04.023

7. I. Gravalos, N. Ziakas, S. Loutridis, Th. Gialamas, A mechatronic system for automated topping and suckering of tobacco plants, Computers and Electronics in Agriculture 0168-1699, 104986 (2019), https://doi.org/10.1016/j.compag.2019.104986

8. M. Kukla, P. Tarkowski, I. Malujda, K. Talaśka, J. Górecki, Determination of the Torque Characteristics of a Stepper Motor, Procedia Engineering 1877-7058, 375-379 (2016), https://doi.org/10.1016/j.proeng.2016.01.226

9. W. Lapsomthop, N. Wongsirirax, A. Kititeerakol, W. Sawangsri, A Build-Your-Own Three Axis CNC PCB Milling Machine 2214-7853, 24404-24413 (2018), https://doi.org/10.1016/j.matpr.2018.10.236

10. K.M. Lynch, N. Marchuk, M.L.Elwin, Embedded Computing and Mechatronics with the PIC32 978-0-12-420165-1, 191-211 (2015), https://doi.org/10.1016/B978-0-12420165-1.00013-5

11. A.I. Dyshekov, I.G. Smirnov, M.A. Mirzaev, M.A. Shereuzhev, Published Principles of functioning of the autonomous device for weed control for precision agriculture, Published under licence by IOP (2020)

12. D. Ibrahim, Arm-Based Microcontroller Multitasking Projects 978-0-12-821227-1, 1-12 (2020), https://doi.org/10.1016/B978-0-12-821227-1.00001-3

13. O. Bishop, Electronics: A First Course (Third Edition) 978-1-85617-695-8, 111-115 (2011), https://doi.org/10.1016/B978-1-85617-695-8.00029-4

14. J. Tilli, A. Brando, G. Fantoni, Gripping Device for Heavy and Deformable Materials Handling: Concept, Design, Selection and Test, Procedia CIRP 2212-8271, 373-378 (2014), https://doi.org/10.1016/j.procir.2014.03.166

15. D. Ibrahim, Arm-Based Microcontroller Multitasking Projects 978-0-12-821227-1, 271321 (2020), https://doi.org/10.1016/B978-0-12-821227-1.00014-1 